\title{
Sox17 Expression Patterns during Gastrulation and Early Neurulation in the Rabbit Suggest Two Sources of Endoderm Formation
}

\author{
Romia Hassoun Bernd Püschel Christoph Viebahn \\ Abteilung Anatomie und Embryologie, Zentrum Anatomie, Georg-August-Universität, Göttingen, Germany
}

\section{Key Words}

Primitive streak - Epiblast · Anterior marginal crescent • In situ hybridization - Mammalian embryo $\cdot$ Development transient expression at the posterior extremity of the primitive streak suggest that endoderm (possibly hindgut) may be formed close to the emerging cloacal membrane, as well.

Copyright $\odot 2009$ S. Karger AG, Basel

\begin{abstract}
Most gastrointestinal tract and associated gland epithelia originate from the endoderm germ layer discovered by Pander in 1817. The recent surge in stem cell concepts revived interest in the findings of 30 years ago that the endoderm layer itself originates from the epiblast (which since Pander's time had been held to be the forerunner of the ectoderm and mesoderm germ layers only). However, the question as to which parts of the mammalian gastrulation-stage embryonic disc generate endoderm cells is still unresolved. Therefore, the expression of the gene coding for the transcription factor Sox 17, a key transcription factor involved in endoderm formation in mouse, chick, frog, and zebrafish, was analyzed in the rabbit, a model organism for mammalian gastrulation morphology, using whole-mount in situ hybridization and high-resolution histological analysis of embryos at gastrulation and early neurulation stages. Sox 17 mRNA in the mesoderm and lower layer (hypoblast) compartments within and adjacent to Hensen's node and the anterior segment of the primitive streak confirmed the validity of this approach, as this region had previously been shown to form endoderm in mouse and chick. However, Sox17 expression in central and posterior epiblast at pregastrulation stages together with a
\end{abstract}

\section{KARGER}

Fax +4161306 1234

E-Mail karger@karger.ch

www.karger.com (c) 2009 S. Karger AG, Basel

$1422-6405 / 10 / 1912-0068 \$ 26.00 / 0$

Accessible online at:

www.karger.com/cto

\section{Introduction}

In the early vertebrate embryo, the endoderm is classically defined as the ventral germ layer which is the precursor of the epithelial lining of the primitive gut tube and which, during organogenesis, regionalizes along its anterior-posterior and dorsal-ventral axes to give rise to the epithelia of the gastrointestinal tract, the respiratory tract and associated organs such as liver and pancreas as well as thyroid gland and thymus. In amniotes, the evi-

\section{Abbreviations used in this paper}

AMC anterior marginal crescent

AVE anterior visceral endoderm

d.p.c. days post coitum

HMG high mobility group

ISHS in situ hybridisation signal

PGE posterior gastrula extension 
dence that the endoderm (also called 'definitive endoderm') itself is derived from the epiblast and not from the pre-existing primitive endoderm (or hypoblast) of the early embryonic disc is comparatively new (when considering the time that has elapsed since the first description of the endoderm as the 'Schleimschicht' in the chick by [Pander, 1817]). This evidence stems from experiments using mouse blastocyst injection chimeras [Gardner and Rossant, 1979], from tissue potency analysis of the epiblast at the primitive streak stage in rat embryos [LevakSvajger and Svajger, 1974] as well as from the microsurgical grafts of both distal and anterior regions of the mouse embryonic epiblast to ectopic sites at the primitive streak stages [reviewed by Beddington, 1983; Skreb et al., 1976]. Endoderm fate maps were constructed accordingly for mouse [Lawson et al., 1986, 1987, 1991; Tam et al., 2007] and chick [Kimura et al., 2006; Kirby et al., 2003; Lawson and Schoenwolf, 2003] and suggest that extensive cellular movements (similar to those observed in lower vertebrates [frog: Keller, 1975, 1976; Chalmers and Slack, 2000; zebrafish: Warga and Nüsslein-Volhard, 1999] occur in the primitive streak area of the gastrulating embryo and eventually contribute to formation of the endoderm [Lawson and Pedersen, 1992; Parameswaran and Tam, 1995; Quinlan et al., 1995; Lawson, 1999]. These migrating epiblast cells also form the other principal germ layers (ectoderm and mesoderm) and thus establish the whole body plan, which makes for even more complex morphogenetic movements at the cellular level. For example, the progenitors of the gut endoderm are initially localized in the distal regions of the mouse egg cylinder together with mesoderm-forming cells and are, therefore, also called mesendoderm [Beddington, 1981; Tam and Beddington, 1987; Tam, 1989]; these cells are thought to ingress through the anterior segment of the primitive streak 8$10 \mathrm{~h}$ after the onset of gastrulation, i.e. at mid-streak stage, and undergo epithelial-mesenchymal transition. Subsequently, some of these cells relocate to the ventralmost layer of the embryo and form an epithelium again while others retain a mesenchymal character and stay in the mesoderm compartment [Kadokawa et al., 1987; Lawson et al., 1987; Tam and Beddington, 1992]. The newly recruited endoderm cells rapidly expand anteriorly and laterally to displace progressively the pre-existing primitive (or visceral) endoderm to extraembryonic sites [Lawson et al., 1986, 1987; Tam et al., 1993; Lin et al., 1994]. Most recently, however, live imaging experiments carried out in the mouse suggest an even more complex but only partial integration of epiblast-derived cells into visceral endoderm, which is then followed by recruitment of vis-

Sox17 Expression Patterns and Endoderm Formation in the Rabbit ceral endoderm cells to form endoderm-derived tissues [Kwon et al., 2008].

Basic molecular mechanisms involved in endoderm specification have been elucidated in several vertebrate model animals such as frog (Xenopus), zebrafish, chick and mouse. The key zygotic factors in the signaling pathway, which are conserved within these species, are the Nodal-related TGF $\beta$ signaling ligands, the Mix-like family of homeodomain transcription factors, the Gata4/5/6 zinc-finger transcription factors and, finally, the high mobility group (HMG) DNA-binding protein Sox17 [Woodland and Zorn, 2008]. Related to the 'sex-determining gene on the Y chromosome (sry)' sox17 belongs to the Sox subgroup F [Lefebvre et al., 2007] and was originally identified as a stage-specific transcriptional regulator during mouse spermatogenesis [Kanai et al., 1996]. Sox 17 was then implicated in endoderm formation when its orthologs Xsox17 $\alpha$ (and Xsox17 $\beta$ ) and Zsox17 were found to be expressed specifically in the endoderm of Xenopus [Hudson et al., 1997] and zebrafish [Alexander and Stainier, 1999], respectively, and when sox17 was indeed found in the endoderm in a highly dynamic pattern in the mouse [Kinder et al., 2001; Kanai-Azuma et al., 2002]. Interestingly, murine sox17-null cells are able to contribute to the early endoderm along the whole intestinal tube; however, from 8.0 days post coitum (d.p.c.) onwards, increased apoptosis in the foregut and failure of mutant cells to differentiate and proliferate in the posterior gut lead to a depletion of endoderm; furthermore, sox17-null ES cells are unable to compete with the wildtype cells in colonizing the gut endoderm in the chimeras [Kanai-Azuma et al., 2002]. These functional studies thus made sox 17 known as a key downstream regulatory component of the signaling pathway which is involved in endoderm induction and endoderm differentiation.

Whereas a wealth of information on the cellular fate and on signalling pathways regulating endoderm differentiation is thus available, the topographic distribution of endoderm differentiation signals within the tissue compartments of the early embryo is still little studied, although such knowledge may reveal important clues as to the principles of endoderm formation. To find a location for initial endoderm formation and to differentiate between characteristics of primitive and definitive endoderm, we report here on the tissue distribution of sox17 in the rabbit embryo throughout gastrulation, i.e. from pre-streak to early somite stages. This may lead to a prospective sequential map for the time and the exact place of origin of the endoderm in mammals. As the endoderm is a central structure around which the body plan is con- 
structed, a morphogenetic map for the specification of the mammalian germ layers in general may emerge as well.

\section{Materials and Methods}

\section{Animal Tissues}

Uteri of naturally mated New Zealand White rabbits (Lammers, Euskirchen, Germany) were removed through cesarean section after injecting an overdose $(320 \mathrm{mg})$ of Narcoren ${ }^{\circledR}(\mathrm{Me}-$ rial, Halbergmoos, Germany) intravenously. Blastocysts at 6.256.75 d.p.c. were flushed using warm sterile phosphate-buffered saline (PBS) and then washed in warm PBS twice to remove blood and cellular residue. Older embryos (7.0-8.0 d.p.c.) were dissected from uteri suspended in sterile PBS using iridectomy scissors after opening the uterine wall antimesometrially. After flushing or primary dissection embryos were fixed in $4 \%$ paraformaldehyde (PFA) in phosphate buffer for $1 \mathrm{~h}$ at room temperature and dissected using iridectomy scissors or flame-polished tungsten needles to eliminate the coats (zona pellucida equivalents or 'neozona' [Denker, 2000]) and most of the extraembryonic tissue. Embryos were staged using dark-field optics according to the developmental stages 1 to stage 7 as defined in [Viebahn, 2004] and [Blum et al., 2007]. Stages and embryonic age corresponded roughly as follows: 6.25 to 6.75 d.p.c.: stages 1 to $4 ; 7.0$ to 7.6 d.p.c.: stages 5 and 6; 8.0 d.p.c.: stage 7 . The dissected embryonic discs were dehydrated and stored in $100 \%$ ethanol at $-20^{\circ} \mathrm{C}$ until used for in situ hybridization.

\section{In situ Hybridization}

Digoxigenin-labeled mRNA probes were generated using a 707-bp mouse Sox17 cDNA (kind gift of Dr. H. Lickert, München, Germany), which spans the coding region from nucleotides No. 1102 through to 1808 of the mouse sox 17 cDNA (GenBank accession No. NM_011441), and chemocompetent cells (DH5alpha Escherichia coli cells, Invitrogen, Karlsruhe, Germany) and chemicals (Roche, Mannheim, Germany or Merck, Darmstadt, Germany) as described [Weisheit et al., 2002]. In situ hybridization procedure was carried out using a standard protocol [Lowe et al., 1996; Belo et al., 1997], which had been adapted for the requirements of early rabbit embryonic discs [Weisheit et al., 2002]. As a first step the stored embryonic discs were transferred to nylon baskets under strictly sterile conditions, rehydrated and treated with proteinase $\mathrm{K}[10 \mu \mathrm{g} / \mathrm{ml}$ proteinase $\mathrm{K}$ in PBT (PBS containing $0.1 \%$ Tween 20; Sigma, München, Germany)] at room temperature for different periods of time according to their stage of development as follows: $1 \mathrm{~min}$ for stage $0 ; 2 \mathrm{~min}$ for stages 1 and 2; 3 min for stage 3; 5 min for stage 4 embryos [Idkowiak et al., 2004]; 10 min for stage 5; 15 min for stage 6 and 20 min for stage 7 embryos; next, all embryos were fixed for $20 \mathrm{~min}$ in $0.2 \%$ glutaraldehyde/PBT. Hybridization buffer consisted of 50\% formamide, $1.4 \times$ SSC (pH 4.5), $0.5 \mathrm{~mm}$ EDTA, $50 \mu \mathrm{g} / \mathrm{ml} \mathrm{t}-\mathrm{RNA}, 0.2 \%$ Tween 20, 0.5\% CHAPS and $50 \mu \mathrm{g} / \mathrm{ml}$ heparin (Sigma). During the pre-hybridization $\left(70^{\circ} \mathrm{C}\right.$ for $1 \mathrm{~h}$ in the hybridization buffer) and hybridization processes $\left(70^{\circ} \mathrm{C}\right.$ overnight in the hybridization buffer with $1 \mu \mathrm{l}$ cRNA previously denatured at $95^{\circ} \mathrm{C}$ for $5 \mathrm{~min}$ ) the baskets containing the embryonic discs were transferred to sterile screw-top PVC tubes (Bibby-Sterilin, Staffordshire, UK).
To remove the unbound cRNA, the embryos were washed in hybridization buffer and MABT (100 mM maleic acid, $150 \mathrm{~mm} \mathrm{NaCl}$, $0.1 \%$ Tween $20, \mathrm{pH}$ 7.5). To block nonspecific antibody-binding sites, the embryos were incubated in MABT with 2\% Roche blocking reagent and $20 \%$ heat-inactivated goat serum; hybridized RNA was visualized using anti-digoxigenin antibody coupled to alkaline phosphatase and BM-purple substrate (both Roche, Mannheim, Germany). Finally, embryos were removed from the nylon baskets and transferred to Petri dishes filled with the substrate to initiate the color reaction, which was then allowed to proceed at room temperature in the dark to exhaustion of the alkaline phosphatase enzyme, i.e. until no further accumulation of the bluish BM-purple reaction product (which started to appear on the second day of BM-purple incubation) was observed in the stereo microscope at high magnification (usually within 4-9 days).

\section{Microscopy}

To record the gross morphological distribution of the reaction, the stained embryos were mounted in Mowiol4-88 (Hoechst, Frankfurt, Germany) under a cover glass and photographed prior to dehydrating in ethanol and embedding in Technovit $8100^{\circledR}$ (Heraeus-Kulzer, Werheim, Germany) at $4{ }^{\circ} \mathrm{C}$ in tablet moulds as described [Idkowiak et al., 2004]. Polymerized blocks were trimmed and serially sectioned using glass knives at $5 \mu \mathrm{m}$ thickness in the sagittal or transverse plane. After the position of a given serial section had been verified in the photographs of the gross morphological overview at low magnification, the allocation of sox17-expressing cells to the topography of the embryo and, within that location, to tissue layers was determined at high magnification. In most cases, i.e. for sections with tissues which contained strong in situ hybridization reactions, photographs were taken using the differential interference contrast (DIC) setting of the microscope (Axioskop, Zeiss, Göttingen, Germany) to visualize the morphology of the parts of the section where no in situ hybridization reaction had occurred. For tissues with exclusively very weak reactions, DIC was not used; this ensured that the intensity of the reaction was not obscured by the contrast enhancement in the surrounding nonstained cells (fig. $2 \mathrm{~N}, \mathrm{~N}^{\prime}$ ).

\section{Results}

All in situ hybridization experiments carried out as described above typically produce well-defined BM purple staining reactions in a variety of cell and tissue types at every stage analyzed in this study: In most cases, these in situ hybridization signals (ISHSs) start to occur within 1 day after the beginning of the final step of the in situ hybridization procedure and they have a high signal-tonoise ratio, i.e. staining is confined to the cytoplasmic domain of a given cell and strongly stained cells frequently lie next to unstained cells or cell groups. ISHSs with a reaction intensity between these extremes also occur frequently and are referred to as weak ISHSs in the following 
Fig. 1. Sox17 expression patterns in rabbit embryo at stages 1 and 2 (6.25 d.p.c.) as determined by in situ hybridization analyses in en face views (A, B) and 5- $\mu$ m sagittal sections at low (C, D) and high magnification (E-H). The anterior border of the embryonic disc is marked by asterisks in $\mathbf{A}$. Insets in $\mathbf{A}$ and $\mathbf{B}$ highlight the morphological characteristics of stages 1 and 2, respectively. All sections are orientated with the anterior end to the left and the epiblast to the top. The borders of the embryonic discs in the sections are defined by asterisks. Bars and letters refer to the positions of the sections; boxes in $\mathbf{C}$ and $\mathbf{D}$ indicate to the positions of the high magnification details shown in $\mathbf{E}-\mathbf{H}$. amc = Anterior marginal crescent, $\mathrm{ep}=$ epiblast, $\mathrm{h}=$ hypoblast, pge $=$ posterior gastrula extension, $\operatorname{trb}=$ trophoblast, yse = yolk sac epithelium; arrowhead in $\mathbf{F}$ points to a labeled hypoblast cell. Scale bar: A, B $80 \mu \mathrm{m}$; C, D $50 \mu \mathrm{m}$; E-H $13 \mu \mathrm{m}$.

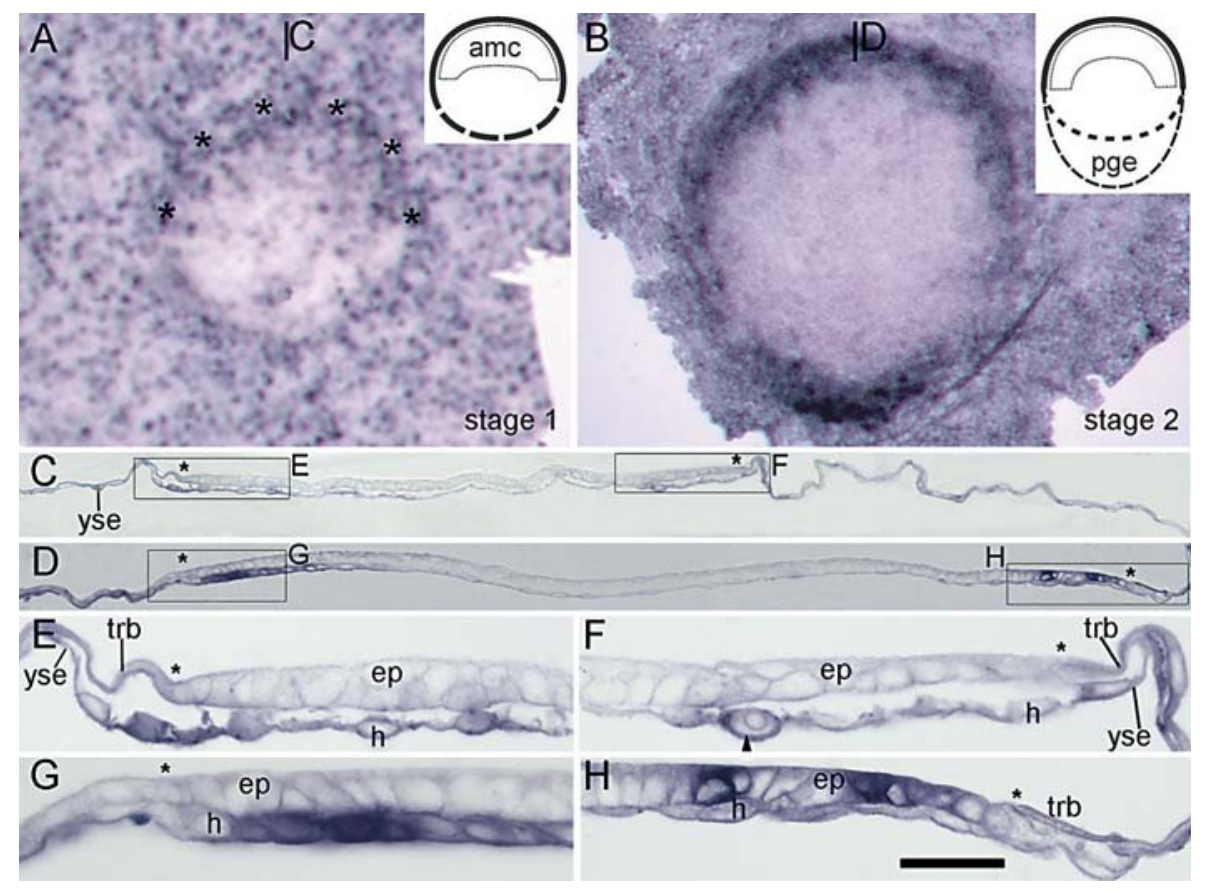

description. As reactions occur in a variety of different cells, tissues and organ anlagen, results are presented here stage by stage rather than by following up the development of a particular organ anlage, for example.

\section{Pre-Streak Stages (Stages 1 and 2)}

At stage 1, the embryonic disc can be recognized - if viewed en face under dark-field illumination - on the surface of the blastocyst as an area of high cellular density. One side of the embryonic disc features a stretch with a relatively sharp contour, while the opposite side of the disc displays an irregular, ragged, and therefore indistinct contour; the distinct contour marks the anterior marginal crescent (AMC) as the earliest axial structure at the anterior pole of the mammalian embryonic disc (fig. 1A, inset). Histologically, the AMC is characterized by increased cellular density and cellular height in both the epiblast and the hypoblast - the two cell layers present in the embryonic disc at this stage - if compared with the posterior margin of the disc (fig. 1E, F).

In dorsal views of stage 1 , a punctate, salt-and-pepper distribution of strong ISHSs with no apparent asymmetry is found in all extraembryonic areas in the vicinity of, and further away from, the embryonic disc (fig. 1A). The disc itself can be recognized as an area with fewer ISHSs in one half and more numerous ISHSs in the other half of the disc. Denser packing of ISHSs is seen in the area co- inciding with the AMC (histologically confirmed, see below), while many small patches with weak or no ISHSs appear in that part of the embryonic disc which can be confirmed histologically to be the posterior half.

In sagittal sections many of the single strongly stained cells are seen to belong to the hypoblast layer in the anterior half of the embryonic disc (fig. 1C, E); within the hypoblast cells ISHSs are mainly found to lie close to the nucleus. A few strongly stained hypoblast cells are also found in the posterior half of the embryonic disc (arrowhead in fig. 1F), and these cells are intermingled with weakly or unstained ones. Some cells in the epiblast layer of the AMC are weakly stained, while others are not stained (fig. 1E); in the posterior half of the embryonic disc the epiblast layer is weakly stained near the posterior border (fig. $1 \mathrm{~F}$ ) but it is unstained further anteriorly, i.e. within the central zone of the embryonic disc (fig. 1C). The punctate pattern in the extraembryonic tissue is found to be generated by cells in the lower layer, i.e. by the yolk sac epithelium cells (fig. 1C, F), whereas weak ISHSs are found in both the intervening yolk sac epithelium cells and in the upper layer, i.e. trophoblast cells (fig. 1C).

At stage 2, a few hours prior to overt primitive streak formation, the anterior contour of the oval-shaped embryonic disc is, in dark-field views, still distinct as before but a sickle-shaped area of low cellular density - called 

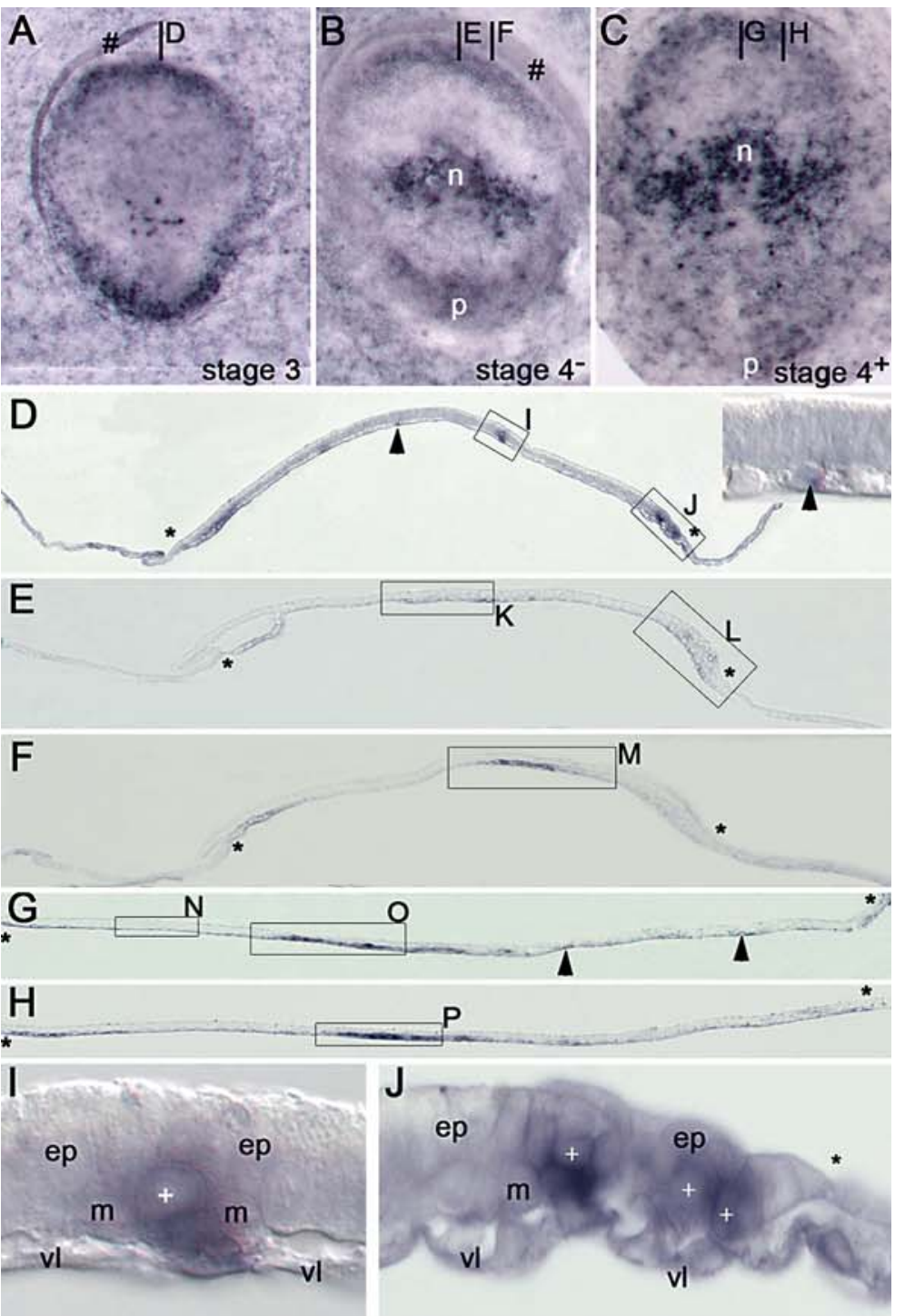

Fig. 2. Distribution of sox17 ISHSs during early gastrulation stages (stages 3-4 at $6.5-6.75$ d.p.c.) as seen in en face views (A-C) and $5-\mu \mathrm{m}$ sagittal sections at low (D-H) and high magnification (I-P). All sections are orientated with the anterior end to the left and the epiblast to the top. The borders of the embryonic discs in the sections are defined by asterisks. Bars and letters refer to the positions of the sections; boxes in $\mathbf{D}-\mathbf{H}$ indicate to the positions of the high magnification details shown in I-P. In $\mathbf{N}^{\prime}$ differential interference contrast (DIC) setting of the magnified details seen in $\mathbf{N}$. D, I, $\mathbf{O}$ and $\mathbf{P}$ are also with DIC setting. ep = Epiblast, $\mathrm{ep}^{\prime}=$ unstained epiblast, $\mathrm{m}=$ mesoderm, $\mathrm{n}=$ Hensen's node, $\mathrm{p}=$ posterior extremity of the primitive streak, $\mathrm{ps}=$ primitive streak, $\mathrm{pm}=$ prechordal mesoderm, vl = ventral layer; + = mesodermal compartment, arrowheads in $\mathbf{D}$ (see also inset in $\mathbf{D}$ ) and $\mathbf{G}$ point to labelled cells in the ventral layer, \# refers to tissue processing artefact. Scale bar: C, E, F $108 \mu \mathrm{m}$; A, B $80 \mu \mathrm{m}$; D, G, H $50 \mu \mathrm{m}$; I-P $13 \mu \mathrm{m}$.
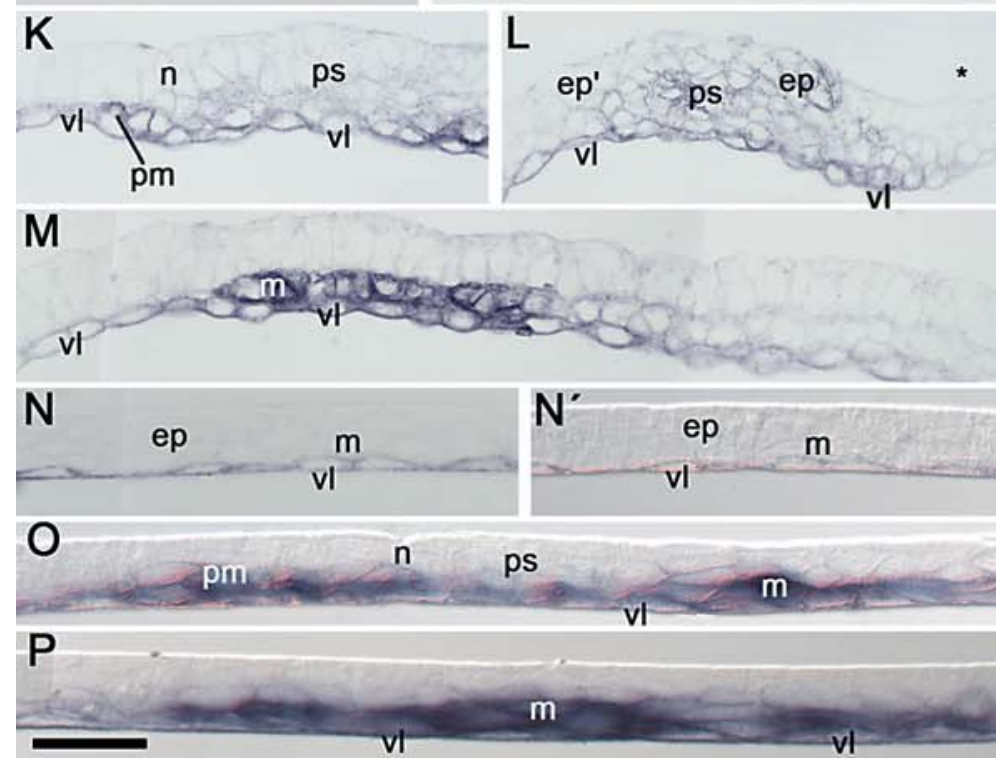
the posterior gastrula extension (PGE) - appears within the posterior half of the embryonic disc and next to the indistinct posterior contour of the embryonic disc (fig. 1B, inset). Sox17 ISHSs of stage 2 embryos are still strong but produce less of a salt-and-pepper appearance and are confined to the periphery of both the AMC and the PGE (fig. 1B); only close to the posterior pole of the embryonic disc, i.e. in the central segment of the posterior contour, some single strong ISHSs arise. The other parts of the embryonic disc show weak reactions and are devoid of the dispersed strong ISHSs seen at stage 1. In extraembryonic areas sox17 ISHSs are for the most part evenly distributed at this stage.

In sagittal sections of this stage, strong ISHSs are found in hypoblast cells of the AMC domain, and these cells, which lie now close to each other and are up to twice as high when compared with those at the opposite side (fig. $1 \mathrm{G}, \mathrm{H}$ ), are located posterior and adjacent to 1-3 rows of hypoblast cells with only weak or no reaction within the anterior border of the embryonic disc (fig. 1G). However, the reactions in epiblast cells of this region are similar to those at the previous stage (fig. 1E). The cells with strong reaction seen in en face views in the central segment of the peripheral PGE portion belong to the epiblast layer, which can be distinguished by their cuboidal shape from adjacent (extraembryonic) squamous trophoblast cells (fig. 1F, H); these epiblast cells, in which strong ISHSs fill most of the cytoplasmic rim around the nucleus, lie mainly isolated or in small groups amongst several neighbouring epiblast cells showing no or only weak ISHSs; this produces the mosaic-like staining pattern in the epiblast seen at the posterior pole of the embryonic disc in dorsal views (fig. 1B); hypoblast cells in this area, on the other hand, are only weakly stained (fig. 1H). Other peripheral segments of the PGE show weak ISHSs in both epiblast and hypoblast layers (not shown). The central area of the embryonic disc shows weak ISHSs in the hypoblast layer (fig. 1D); extraembryonic tissues exhibit weak reactions in both the yolk sac epithelium and in the trophoblast layer (fig. 1D, left).

\section{Gastrulation Stages (Stages 3 and 4)}

The gastrulation process becomes apparent at stage 3, when the primitive streak can be seen as a localized cellular density stretching along the posterior half of the midline in en face views under dark-field illumination. Thus, the embryonic disc has elongated towards the posterior pole and its previous oval shape is now transformed into a pear-like shape. The density of the streak is caused by cellular accumulation, following extensive cellular movements within the epiblast directed towards the posterior midline, and provides the basis for the ingression of epiblast cells through a narrow discontinuity in the basement membrane stretching along the primitive streak. Functional studies in mouse and chick (see 'Introduction') suggest that endoderm cells are inserted into the ventral layer of the embryonic disc, which prior to this stage consists of hypoblast cells only. Therefore, from the start of gastrulation onwards, there are likely to be two cell populations in the ventral layer of the embryonic disc. As no reliable markers exist to date to distinguish endoderm from hypoblast, the following description of the cell layers uses 'ventral layer' instead of 'hypoblast' for most areas of the embryonic disc from stage 3 onwards; only for the AMC, where endoderm is inserted at later stages, is the ventral layer still addressed as hypoblast until stage 4 .

In dorsal views of stage 3 embryos, strong sox 17 ISHSs in the AMC and in the posterior parts of PGE lie close to the border of the embryonic disc, similar to those at stage 2 (fig. 2A); however, due to the elongation of the disc these anterior and posterior domains are now more clearly separated by a peripheral band of weakly labeled cells on both sides of the embryonic disc. In addition, single strong ISHSs appear as isolated spots near the anterior midline of the posterior half of the embryonic disc; these spots lie just anterior to the PGE and coincide roughly with the anterior extremity of the emerging primitive streak. Apart from these strongly stained parts of the embryonic disc the remainder of the embryonic disc shows weak reactions only. The extraembryonic tissues exhibit staining patterns similar to those seen at the previous stage.

Sagittal sections of this stage show principally the same staining patterns in the hypoblast found at stage 2 anteriorly and posteriorly (fig. 1G, H, fig. 2D, J, respectively); in addition, single stained cells lie amongst unstained ones in the ventral layer of the anterior half of the embryonic disc (arrowheads in fig. 2D and inset). In the epiblast of the posterior part of PGE, i.e. close to the posterior disc border, the mosaic pattern can be seen similar to that at stage 2, but now ISHSs lie either in the epiblast or in the mesoderm compartments (fig. 2J). The single strongly stained cells seen near the anterior midline of the posterior half of the embryonic disc are also found to lie in the mesoderm compartment, i.e. in the rostral part of the primitive streak (fig. 2I). Generally, there are now weak reactions in the basal parts of the epiblast layer throughout the whole embryonic disc (fig. 2I, J). The ISHSs in the extraembryonic tissues are weak in the tro- 
phoblast cells and in the yolk sac epithelium cells (fig. 2D).

As gastrulation proceeds to stage 4 , the overview of the whole-mount embryo shows that the embryonic disc elongates further and the Hensen's node appears as a cellular density at the anterior extremity of the primitive streak. As a sign of the establishment of Hensen's node, the prechordal mesoderm cells emerge from Hensen's node ('pm' in fig. $2 \mathrm{~K}$ ) and migrate anteriorly to presage the formation of notochordal process (stage 5, fig. 3A, B).

In dorsal views of early Hensen's node stages (stage $4^{-}$, fig. $2 \mathrm{~B}$ ), both the AMC domain and the domain near the posterior border of the embryonic disc still present sox17 ISHSs, but these are weaker than before, and in the area where the single strongly stained spots are seen near the anterior part of the primitive streak at the previous stage (stage 3), a horizontally oriented strongly stained domain arises on either side of the anterior segment of the primitive streak (fig. 2B). In the extraembryonic tissues the intensity of the reactions is only weak or absent at this stage.

Sagittal sections show that the hypoblast layer of the AMC area continues to have ISHSs whereas no ISHSs are found in the epiblast layer (fig. 2E, F). Weak ISHSs can be recognized in the ventral layer covering the primitive streak (fig. $2 \mathrm{~K}, \mathrm{~L}$ ) and in the ventral layer stretching between Hensen's node and the anterior margin of the embryonic disc (fig. 2E); in fact, in the anterior part of the primitive streak mesoderm cells, including the first prechordal mesoderm cells emanating anteriorly from Hensen's node, are also weakly stained (fig. $2 \mathrm{~K}$ ), whereas in the posterior extremity of the primitive streak they are weakly stained in all three layers (fig. 2L). Lateral to the anterior segment of the expanded primitive streak, the continuous bands of strong ISHSs appear in both mesoderm and ventral layer cells (fig. 2M). The extraembryonic tissues show only weak reactions in the yolk sac epithelium cells at this stage (fig. 2E, F).

In dorsal views of late stage 4 embryos (fig. $2 \mathrm{C}$ ), the anterior margin of the embryonic disc still carries some ISHSs within a mostly weak anterior sox 17 expression domain. The strongly stained transverse domain seen in the last stage expands anteromedially and posterolaterally thereby now encompassing Hensen's node and more of the anterior segment of the primitive streak than before. Weak sox17 ISHSs are found in the area located between the anterior margin of the embryonic disc and this strongly stained transverse domain, whereas the area located posterior to the strongly stained transverse domain, in- cluding the primitive streak, displays weak reactions intermingled with moderately strong ISHSs. The extraembryonic tissues are stained weakly.

Sections of this stage show the strong ISHSs seen in the transverse domain (fig. $2 \mathrm{H}$ ) to lie in the mesoderm compartment juxtaposed to the ventral layer; these cells intermingle with weakly stained cells located in both the mesoderm and ventral layer compartments and make this transverse expression domain less compact than at the early stage 4 . Only few strongly stained cells lie in the ventral layer covering this domain (fig. 2P). In the midline, strong ISHSs are observed in the prechordal mesoderm cells that have emerged from Hensen's node, and weak reactions are found in the ventral layer cells covering these prechordal mesoderm cells (fig. 2O). In the anterior part of the primitive streak, some strongly stained cells in the mesoderm compartments are located adjacent to the ventral layer and are found to lie next to weakly stained cells within the mesoderm and the ventral layers (fig. 2O). Throughout the other parts of the primitive streak, single strongly stained cells are found in the ventral layer (arrowheads in fig. 2G), whereas the posterior extremity of the primitive streak still shows weak ISHSs in all three layers similar to the situation seen at stage $4^{-}$ (fig. 2L). At the anterior margin of the embryonic disc, strong reactions are still present in the hypoblast layer but not in the epiblast layer (fig. 2G, H). The weak reactions located in the region between the anterior margin of the embryonic disc and the strongly stained transverse domain are found to lie in the ventral layer, as can be seen particularly well if no differential interference contrast is used for light microscopic analysis (fig. $2 \mathrm{~N}$ and $\mathrm{N}^{\prime}$ ). Except for the weak reactions found in the epiblast of the posterior extremity of the primitive streak, the epiblast layer throughout the whole embryo shows no ISHSs (fig. 2G, H). Reactions are weak in most of the yolk sac epithelium cells, whereas the upper layer of the extraembryonic tissues (trophoblast) shows no ISHSs.

\section{Neurulation Stages (Stages 5, 6 and 7)}

As a result of gastrulation the notochordal process begins to emerge from Hensen's node along the anterior midline of the embryonic disc (fig. 3A) and, in parallel, the anterior half of embryonic disc begins to elongate along the anterior-posterior axis to accommodate the neural plate as the first step of neurulation. The increasing length of the notochordal process can be determined easily in dorsal views during early neurulation and is, therefore, used here to divide the beginning of neurulation into 3 stages: Stage 5 covers the period after the first 
prechordal mesoderm cells leave Hensen's node (end of stage 4) until the notochord acquires the same length as the primitive streak (fig. 3B); during stage 6, the notochordal process elongates further and its posterior part becomes nearly twice as wide as its anterior part; this is in contrast to the posterior half of the embryonic disc which becomes narrower than its anterior half (fig. 4A, B), and at stage 7, the first somites start to form on either side about equidistantly from the two extremities of the notochordal process, i.e. next to the anterior extremity of the posterior, wide part of the notochordal process (fig. 4C, D).

In dorsal views of early stage 5 embryos (fig. $3 \mathrm{~A}$ ), the strongest ISHSs are found on both sides of the (short) notochordal process and both near the anterior margin of the embryonic disc and at the posterior extremity of the primitive streak. The domain next to the notochordal process is similar in shape to the transverse domain observed first at stage $4^{-}$(fig. $2 \mathrm{~B}$ ) and a little more expanded than at stage $4^{+}$(fig. $2 \mathrm{C}$ ); the domain still encompasses the anterior part of the primitive streak and Hensen's node and, therefore, has broadened along the anteriorposterior axis in comparison to earlier stages. In the anterior half of the primitive streak itself, ISHSs are weak; weaker still are ISHSs in Hensen's node and notochordal process and in the posterior third of the embryonic disc. The borders of the embryonic disc encompassing the posterior parts of the primitive streak show, at this stage, also weak ISHSs except for the posterior-most extremity of the primitive streak which is still marked with strong ISHSs. In the extraembryonic tissues, weak ISHSs are distributed and intermingled with small unstained areas.

In sagittal sections of early stage 5 embryos, the strong ISHSs seen in the overview lateral to the notochordal process and primitive streak are found to be located in both the mesoderm compartment and in the ventral layer covering this domain (fig. $3 \mathrm{H}$ ); towards the anterior margin this domain of strongly stained cells is continuous laterally with the domain of strongly stained cells in the hypoblast and some overlying mesoderm cells (fig. 3D, H). In the midline, the transverse domain is interrupted by the unstained notochordal process (fig. $3 \mathrm{~F}$ ) but it is continuous across the midline anterior to the tip of the notochordal process due to the weak ISHSs in the prechordal mesoderm cells and in the ventral layer covering the prechordal mesoderm (fig. 3F). However, the ventral layer covering the early notochordal process and Hensen's node also shows weak ISHSs (fig. 3F). Along the primitive streak, weak ISHSs are found in some cells located in the mesoderm and ventral layers (fig. 3C, F), but the poste-

Sox17 Expression Patterns and Endoderm Formation in the Rabbit rior extremity of the primitive streak shows weak reactions in a continuous oblique band of cells lying across all three layers, and this band touches anteroventrally several more strongly stained cells in the ventral layer underlying the primitive streak (fig. $3 \mathrm{G}$ ). The reactions in the extraembryonic tissues are found to be weak in most yolk sac epithelium cells and absent in the trophoblast cells (not shown).

In dorsal views of late stage 5 embryos (fig. 3B), two bands of strong reactions are found to run parallel on each side of the elongating notochordal process. At the level coinciding roughly with Hensen's node, these bands extend posterolaterally to contact the posterolateral margins of the embryonic disc. Strong ISHSs are found along almost all margins of the embryonic disc except for the posterior pole where the margin shows only weak ISHSs. Weak ISHSs are found in an arch-like region located between the strongly stained parallel bands and the anterior and lateral strongly stained margins (crosses in fig. 3B). No ISHSs are found in Hensen's node and notochordal process. In the primitive streak, weak reactions are found in the anterior segment and in the posterior extremity, whereas most of the posterior half shows no reactions. In the extraembryonic tissues most Sox 17 ISHSs are found next to the embryonic disc, while the remaining extraembryonic areas have weak or no ISHSs.

Sections analyzed for this stage show that in the bandlike domains of ISHSs located next to the notochordal process reactions are strongest in the ventral layer, but weaker in the mesoderm and weaker still in the neural plate overlying them (fig. 3J). In the oblique bands located laterally at the level of Hensen's node and most of the primitive streak, reactions are confined to the ventral layer (fig. 3K-M). Cells of the notochordal process do not show ISHSs and they are separated from the strongly stained ventral layer cells laterally by a few cells which are unstained and difficult to allocate either to the notochordal process or the ventral layer laterally (arrowheads in fig. 3J). No ISHSs are found in the prechordal mesoderm cells next to weak reactions in the mesoderm and ectoderm (fig. 3I). Similarly, Hensen's node and the primitive streak are devoid of ISHSs, while the ventral layer covering these parts has no ISHSs at the level of Hensen's node (fig. 3K) and also the level of the most posterior half of the primitive streak (fig. 3M), whereas stronger ISHSs in this layer are found at both levels of the anterior part and posterior extremity of the primitive streak (fig. 3L and $\mathrm{N}$, respectively). At the margins of the embryo including the posterolateral margins encom- 


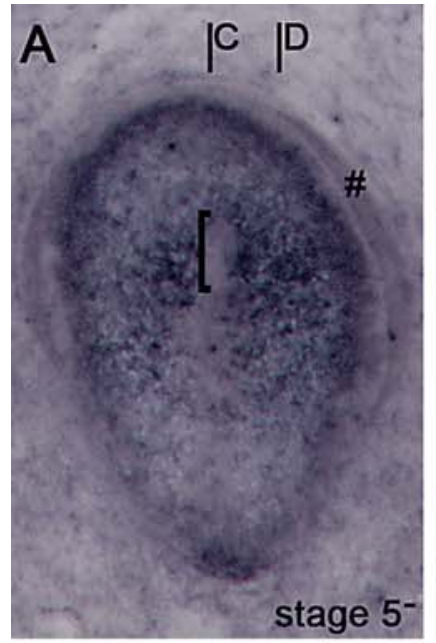

E

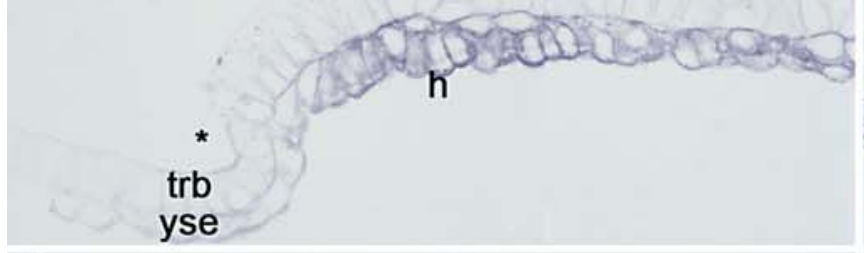

G

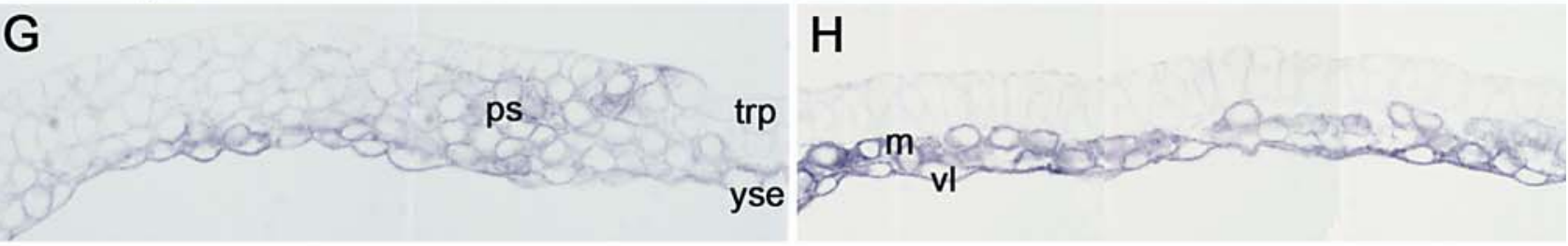


passing the posterior half of the embryonic disc, which are strongly stained at this stage, strong ISHSs are found in the ventral layer and weak ones in mesoderm cells (fig. 3I). In the arch-like area located between the lateral border of the embryo and the band-like domain (area marked ' $x$ ' in fig. 3B), the ISHSs are weak to absent in the mesoderm and in the ventral layer posteriorly (fig. 3J, right) and are weak in the mesoderm but stronger in the ventral layer more anteriorly (fig. 3I). Strong ISHSs are found in the yolk sac epithelium of the extraembryonic tissues adjacent to the embryo while in these cells further away from the embryo the reactions are weak or absent, the trophoblast of the extraembryonic tissues shows no ISHSs (fig. 3I).

In dorsal views of early stage 6 embryos (fig. 4A), the two oblique bands of strong ISHSs are found again at each side of the most anterior part of the wide, posterior region of the notochord and these bands extend posterolaterally to contact the margins of the embryo, a situation very similar to that found at stage $5^{+}$(fig. 3B). However, distinctly weaker ISHSs are found in two longitudinal band-like domains on either side of the anterior, narrow part of the notochordal process and extend the oblique bands of strong ISHSs towards the anterior pole of the embryo. Strong ISHSs are found in most of the embryonic margins except for the border encompassing the posterior pole of the embryonic disc, which shows no reactions. In an arch-like area located between the strongly stained anterior margin of the embryo and the weakly stained two bands, which run along the anterior narrow part of the notochordal process, no or weak ISHSs are

Fig. 3. Sox 17 expression patterns during early neurulation stages (stages $5^{-}$at 7.0 d.p.c. and $5^{+}$at 7.6 d.p.c.) as seen in en face views (A, B) and 5- $\mu \mathrm{m}$ sections at low (C, D) and high magnification (E-N). All sections are orientated with the epiblast to the top and the sagittal ones are orientated with the anterior end to the left and the epiblast to the top. The borders of the embryonic discs in the sections are defined by asterisks. Bars and letters in $\mathbf{A}$ refer to the positions of the sagittal sections and in $\mathbf{B}$ according to the length of the transverse sections shown in $\mathbf{I - N}$; boxes in $\mathbf{C}, \mathbf{D}$ indicate to the positions of the high magnification details shown in $\mathbf{E}-\mathbf{H}$. I-N are with differential interference contrast (DIC) settings. $\mathrm{h}=$ Hypoblast, $\mathrm{m}=$ mesoderm, $\mathrm{n}=$ Hensen's node, $\mathrm{nc}=$ notochordal process, $\mathrm{np}=$ neural plate, $\mathrm{pm}=$ prechordal mesoderm, $\mathrm{ps}=$ primitive streak, $\mathrm{trb}=$ trophoblast, $\mathrm{vl}=$ ventral layer, yse $=$ yolk sac epithelium; arrowheads in $\mathbf{J}$ indicate to unstained cells in the ventral layer located closed to the notochordal process, \# refers to tissue fold artefact, brackets in $\mathbf{A}$ and $\mathbf{B}$ mark the length of the notochordal process, $\mathrm{x}$ marks arch-like weakly stained areas. Scale bar: A, B $108 \mu \mathrm{m}$; C, D $50 \mu \mathrm{m}$; E-N $13 \mu \mathrm{m}$.

Sox17 Expression Patterns and Endoderm Formation in the Rabbit found. No ISHSs are found in Hensen's node and notochordal process. Weak ISHSs can be seen in most of the anterior part of the primitive streak. In the extraembryonic tissues, strong ISHSs are restricted to a narrow band located next to the anterior circumference of the embryonic disc.

In the sections (data not shown), distribution of ISHSs in the strongly stained oblique band-like domains to the germ layers is similar to that of stage 5 (fig. 3J). However, in the weakly stained band-like domains on either side of the anterior, narrow part of the notochord, the ISHSs are found to be weak and in the ventral layer only. The expression pattern in the margins of the embryo is similar to the pattern found at the previous stage (fig. 3I), and - as also found in the last stage - the prechordal mesoderm cells, notochordal process and node show no reactions. The weak reaction found in the overview in most of the anterior part of the primitive streak is found to be confined to the ventral layer (fig. 3L). Strong ISHSs are found in the yolk sac epithelium cells of the extraembryonic tissues adjacent to the embryo, while in the cells further away from the embryo the sox 17 ISHSs are absent; in the trophoblast layer no ISHSs are seen.

At late stage 6, i.e. in the late presomite stage (fig. 4B), the patterns of sox17 ISHSs are found to be similar in form to that of early stage 6; however, the two weakly stained band-like domains on either side of the anterior narrow part of the notochordal process now have strong ISHSs in the ventral layer and weaker ones in the mesoderm layer (see below stage 7; fig. $4 \mathrm{~K}$ ) and are slightly wider than at early stage 6 , while the anterior weakly or non-stained arch-like domain has acquired the shape of an inverted $U$ at this stage. Here, too, the reactions are confined to the mesoderm and the ventral layer. Other than that the distribution of ISHSs is similar to that found at early stage 6 .

At stage 7, when 2-4 somite pairs have formed, the distribution of ISHSs has, again, changed only little as compared to stage 6 . However, the two posterior extremities of the inverted U-shaped anterior domain have widened posteriorly, so that two lateral oval-like areas appear containing a short and narrow longitudinal band of weak ISHSs which coincide with the bilateral heart anlagen just anterior to the level of the most anterior somite (arrowhead in fig. 4C, fig. 4D). Also, the posterior area without ISHSs overlying the posterior half of the primitive streak has turned into an arch-like shape encompassing the weakly stained anterior half of the primitive streak (fig. 4C, D). In the extraembryonic tissues, scattered ISHSs are found again at this stage.

Cells Tissues Organs 2010;191:68-83 


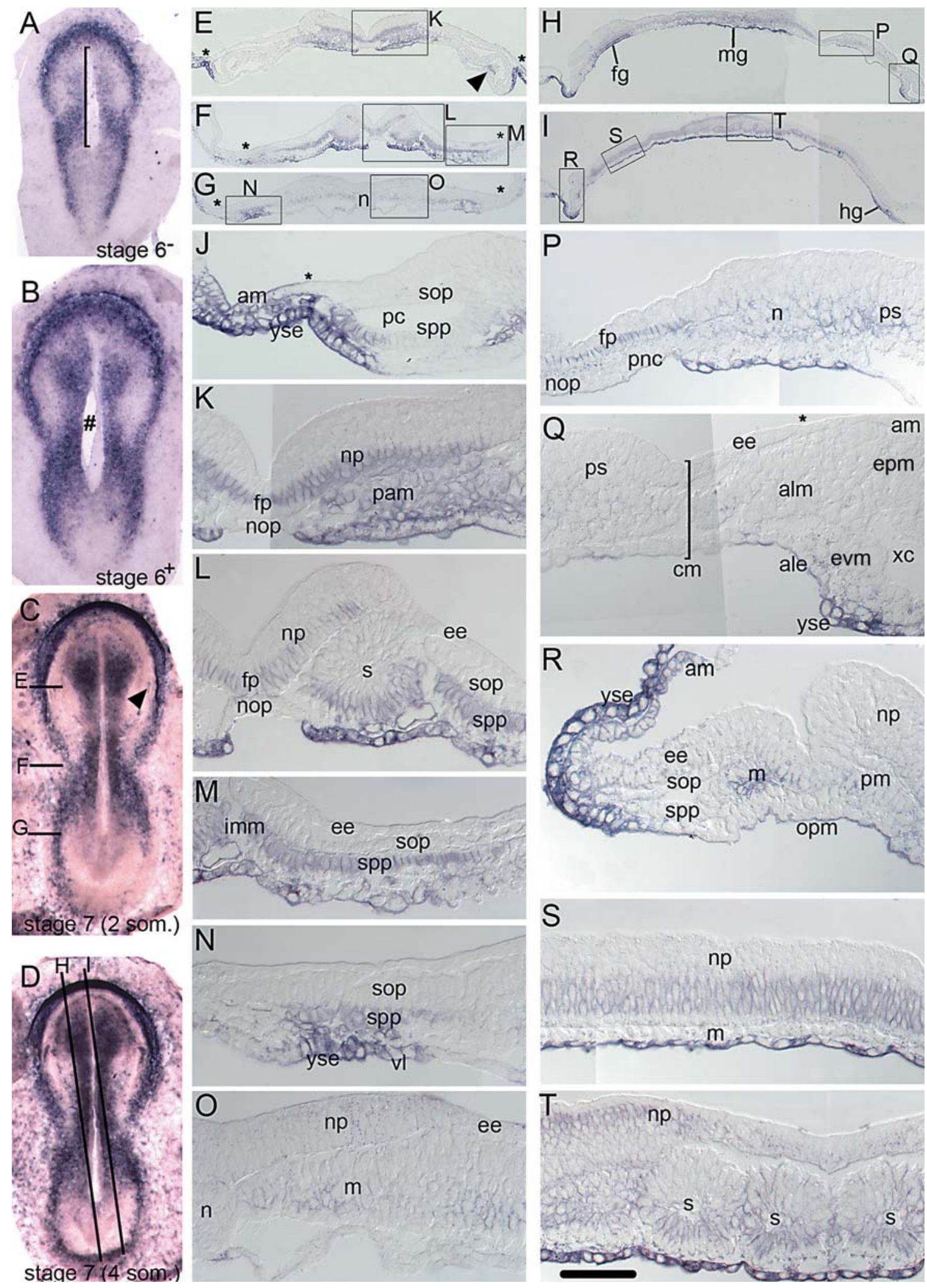

Cells Tissues Organs 2010;191:68-83

Hassoun/Püschel/Viebahn 
The sections obtained at this stage show that the two oblique band-like domains on either side of the posterior notochordal process contain strong ISHSs in the ventral layer (the future midgut at this level, fig. 4H, I). Weak expression is found in the splanchnopleuric mesoderm, which is now separated from the somatopleuric mesoderm by a narrow intraembryonic coelom (fig. 4M, N). At more anterior levels (somite levels: fig. 4F; narrow part of notochordal process: fig. $4 \mathrm{E}$ ), the somites (fig. $4 \mathrm{~L}, \mathrm{~T}$ ) and the neural plate (fig. $4 \mathrm{~K}, \mathrm{~S}$ ), but not the epidermal ectoderm (fig. 4L, M), show weak ISHSs in the ventral half of the epithelium and in the underlying mesoderm; however, strong ISHSs are found in the ventral layer covering these structures (the future foregut, fig. $4 \mathrm{E}, \mathrm{K}, \mathrm{H}, \mathrm{I}$ ). Within the two lateral oval-shaped areas located just anterior to the level of the somites between the strongly stained embryonic disc margins and the strongly stained longitudinal band-like domains, ISHSs are found to lie in the splanchnopleuric mesoderm of the heart anlage and in the underlying ventral layer (future foregut; fig. 4J).

Along the anterior margin, strong reactions are found in the lower layer (hypoblast and yolk sac epithelium) and weak ones in the ectoderm (fig. 4R, J). The two layers of the oropharyngeal membrane show weak or no ISHSs, whereas some splanchnopleuric mesoderm cells anterior and next to this membrane show weak reactions; the pre-

Fig. 4. Sox17 expression patterns during neurulation stages (stages $6^{-}$to 7 at 8.0 d.p.c.) as seen in en face views (A-D) and $5-\mu \mathrm{m}$ sections at low (E-I) and high magnification (J-T). All sections are orientated with the epiblast to the top and the sagittal ones are orientated with the anterior end to the left and the epiblast to the top. Asterisks indicate the borders of the embryonic discs. Bars and letters in $\mathbf{C}$ and $\mathbf{D}$ refer to the positions of transverse and sagittal sections, respectively; boxes in E-I indicate the positions of the high magnification details shown in $\mathbf{K}-\mathbf{T}$, but $\mathbf{J}$ was taken from a section anterior to $\mathbf{E}$. K-T are with differential interference contrast (DIC) settings. ale = Allantoic endoderm, alm = allantoic mesoderm, am = amnion, $\mathrm{cm}=$ emerging cloacal membrane, ee $=$ epidermal ectoderm, epm = extraembryonic parietal mesoderm, evm = extraembryonic visceral mesoderm, $\mathrm{fg}=$ foregut anlage, $\mathrm{fp}=$ floor plate, $\mathrm{hg}=$ hindgut anlage, $\mathrm{imm}=$ intermedial mesoderm, $\mathrm{m}=$ mesoderm, $\mathrm{mg}=$ midgut anlage, $\mathrm{n}=$ Hensen's node, $\mathrm{np}=$ neural plate, nop $=$ notochordal plate, $\mathrm{opm}=$ oropharyngeal membrane, $\mathrm{pc}=$ pericardial cavity, pam $=$ paraxial mesoderm, $\mathrm{pm}=$ prechordal mesoderm, pnc $=$ posterior notochord, $\mathrm{ps}=$ primitive streak, $\mathrm{s}=$ somite, $\mathrm{spp}=$ splanchnopleuric mesoderm, sop = somatopleuric mesoderm, $\mathrm{vl}=$ ventral layer, $\mathrm{xc}=\mathrm{ex}$ traembryonic cavity, yse = yolk sac epithelium; bracket in $\mathbf{A}$ indicates the length of the notochordal process, \# in B refers to tissue damage artefact, arrowheads in $\mathbf{C}$ and $\mathbf{E}$ point to the heart anlage. Scale bar: A-D $156 \mu \mathrm{m}$; H, I $108 \mu \mathrm{m}$; E-G $50 \mu \mathrm{m}$; J-T $13 \mu \mathrm{m}$.

Sox17 Expression Patterns and Endoderm Formation in the Rabbit chordal mesoderm which lies posterior to the oropharyngeal membrane has no or only weak ISHSs (fig. 4R). No ISHSs are found in the notochordal plate (and the posterior notochord), whereas the floor plate of the neuroectoderm directly overlying the notochord shows weak reactions ventrally similar to the weak ISHSs in the ventral parts of neural plate laterally (fig. $4 \mathrm{~K}, \mathrm{P}$ ). Only weak reactions are found in the centre of Hensen's node, whereas in the cells of the ventral layer underlying Hensen's node and the next small part of the primitive streak strong reactions are seen as at earlier stages (fig. 4P). Weak reactions are found in the ectoderm and mesoderm cells lateral to Hensen's node (fig. 4O), whereas the posterior part of the primitive streak located next to the posterior margin of the embryonic disc shows no reactions at this stage (fig. 4Q). Near the posterior margin of the embryonic disc, ISHSs are found in the ventral layer (the future hindgut, fig. 4I, Q), in the allantoic endoderm which is continuous posteriorly with the (strongly stained) yolk sac epithelium (see below) and in the ventral part of the allantoic mesoderm which is continuous posteriorly with the visceral extraembryonic mesoderm. No reactions are found in the amnion and in the parietal extraembryonic mesoderm (fig. 4Q). In the extraembryonic tissues located next to the anterior margin of the embryonic disc, strong ISHSs are found in the yolk sac epithelium and weak ones in the amniotic epithelium (fig. 4I, J, R), whereas the remaining parts of the extraembryonic tissues show weak or strong ISHSs in the yolk sac epithelium intermingled with unstained areas (fig. 4F, G).

\section{Discussion}

This gene expression study shows that the Sox17, as a key transcription factor for vertebrate endoderm formation, displays specific expression patterns which are consistently found throughout the early phases of laying down the body plan from pre- and early gastrulation through to early neurulation stages. Sox17 mRNA is found: (1) in prospective endoderm cells of the central epiblast at the early streak stage, (2) adjacent to the anterior segment of the stage 3 and 4 primitive streak in mesoderm cells and, adjacent to these mesoderm cells, in prospective endoderm cells inserted into the ventral layer, and (3) bilateral to the notochordal process during early neurulation (stage 5), again in both mesoderm and the ventral layer. In addition, and described for the first time in a mammalian embryo, sox $17 \mathrm{mRNA}$ is found in a mosaic-like distribution in the epiblast at the posterior pole 
of the embryonic disc immediately prior to the appearance of mesoderm cells in the primitive streak; this posterior expression domain finds its continuation in all three cell layers at the posterior extremity of the primitive streak as gastrulation proceeds up to early neurulation stages. Possibly indirectly connected with endoderm formation, sox $17 \mathrm{mRNA}$ is also found during early neurulation stages in some mesodermal and neural components lying next to the endoderm as well as in extraembryonic tissues. Apparently without direct connection to locations of endoderm formation, sox 17 mRNA appears in the emerging anterior marginal crescent (AMC), the axis defining structure in the anterior half of the embryonic disc immediately prior to gastrulation, and thereby serves as an additional molecular marker for anterior-posterior axis definition.

The fact that the cRNA probe used in this study was generated from a mouse sox $17 \mathrm{cDNA}$ may be regarded as a methodological limitation, but the distinct expression patterns found here at enhanced stringency conditions $(1.4 \times$ SSC compared to $5 \times$ SSC generally used in ISH; see Materials and Methods) coincide with the known topography of endoderm formation in mouse and chick on the one hand [Lawson et al., 1986; Lawson and Pedersen 1992; Kinder et al., 2001; Kanai-Azuma et al., 2002; Lawson and Schoenwolf, 2003; Pfister et al., 2007] and with hypothetical but topographically plausible additional sites of endoderm formation such as the posterior extremity of the primitive streak, on the other. Together, these two considerations may be taken as providing sufficient support for our main conclusion about the topography of endoderm formation, while further reaching conclusions, e.g. whether other members of the Sox-subgroup F are involved in either of the two endoderm sources suggested here, will require RT-PCR amplification and cloning of a sox $17 \mathrm{cDNA}$ from rabbit using predicted exon sequences from the genomic sequence which became available in the ENSEMBL database only after the experimental part of the study was completed. At present, the positive evidence of the sox17 knock-out mouse [Kanai-Azuma et al., 2002] and the high homology when comparing sox 17 sequences of mouse, rabbit and human (GenBank Accession Nos. NM_011441 [mouse], NM_022454 [human] and ENSEMBL-ID ENSOCUT00000012645 [rabbit]) on the one hand, and the negative evidence that sox18 is most likely not involved in the endoderm cell lineage [Pennisi et al., 2000] on the other, have to be weighed against the probability that our riboprobe (spanning the sox 17 coding region with the high mobility group) recognizes other members of the Sox subgroup F (sox7 and sox18) as well.

The mosaic-like expression pattern seen in the posterior epiblast cells prior to the appearance of the primitive streak has not been found in other mammalian embryos analyzed so far [Kinder et al., 2001; Kanai-Azuma et al., 2002; Pfister et al., 2007] but it is reminiscent of the onset of mesoderm formation, which starts with the specific expression of the key regulatory gene brachyury in single epiblast cells dispersed in a mosaic-like pattern at the posterior pole of the embryonic disc [Viebahn et al., 2002]. Indeed, the consistent finding of this early expression may be an indication for the possibility that the epiblast contains progenitor cells which are primed for endoderm formation simultaneously with, or prior to, mesoderm formation, but extensive experimentation will be needed to find out which of the two progenitor types is a subgroup of the other (in the true sense of the mesendoderm coined for amphibian and fish development), or whether there are, indeed, two separate progenitor populations for endoderm and mesoderm, respectively.

Before the primitive streak acquires the maximal length, another small population of endoderm progenitors seems to appear at its rostral extremity and is in prime position to insert directly into the lower layer, as was observed in the chick at early primitive streak stages and using a newly developed labeling method [Kimura et al., 2007]. However, the relatively low number of early endoderm cells [Tam and Beddington, 1992] may be explained by the second region of endoderm recruitment as defined by posterior sox 17 expression. This interpretation is also supported by the phenotype of the sox 17 knock-out mouse: In this mutant, the severity of the gut phenotype was most pronounced in the posterior gut and sox17-deficient cells were unable to colonize the mid-and hindgut of the chimeras whereas the prospective foregut developed quite normally until the late neural plate stage [Kanai-Azuma et al., 2002]. However, the posterior sox17 expression in the rabbit contrasts with results in mouse and chick, where no sox 17 expression has so far been described at the posterior extremity of the streak [KanaiAzuma et al., 2002; Chapman et al., 2007; Pfister et al., 2007; Tam et al., 2007]. A solution to this discrepancy may lie in a mere difference in the interspecific timing of posterior endoderm commitment and recruitment; this posterior domain may, indeed, contribute precursors of the extraembryonic endoderm (allantoic endoderm and posterior yolk sac endoderm [Tam et al., 2007]), and this process differs profoundly even between closely related mammalian species [Mossman, 1937]. 
As gastrulation proceeds, increased sox 17 expression appears around Hensen's node and the anterior segment of the primitive streak. This coincides with an increasing endoderm contribution described in the same region in the mouse [Kanai-Azuma et al., 2002; Pfister et al., 2007]. In these areas almost all newly recruited cells seem to migrate peripherally some distance within the middle layer and then insert into the ventral layer, probably to form foregut endoderm, a finding also obtained in the chick embryo at similar stages [Kimura et al., 2007]. However, the sox 17 expression domains of the rabbit appear to support differences in endoderm precursor cell movement observed between avian and mammalian embryos, which concern anterior definitive endoderm moving in step with the mesoderm layer [Kimura et al., 2006] in the chick, whereas in the mouse dorsal foregut endoderm moves independently of the mesoderm [Tam et al., 2007].

Not directly related to the endoderm sources described so far, sox17 expression is found in early prechordal mesoderm cells, which indicates that - similar to the situation in the chick [Kimura et al., 2007] - these cells may contribute, after migration anteriorly and laterally, to the foregut. Further anterior to the prechordal mesoderm, there is an expression domain just anterior to the oropharyngeal membrane, and this may be related to the induction process of the very early pancreas primordium because loss of sox 17 function leads to the lack of $P d x 1$ expression (a gene related to pancreas differentiation) while Hex (required for liver differentiation [Bogue et al., 2000]) is still expressed in this area [Stainier, 2002].

Several neural structures such as the neural plate and the floor plate and some mesodermal structures such as somites and splanchnopleuric mesoderm express sox 17 mRNA at early neurulation stages (fig. 4), which may be an indication of sox 17 activity partially overlapping (and co-operating) with sox 2 and sox 3 activity in early neural tissues [Wood and Episkopou, 1999] and, possibly, in the differentiation of the foregut: sox 2 is expressed in the gut endoderm in the chick and mouse, whereas sox 3 transcripts are detected in the posterior region of the foregut in mouse early somite-stage embryos [Wood and Episkopou, 1999] but not in chick embryos [Kimura et al., 2007]. Sox17 is also expressed in developing blood vessels and the heart (fig. 4) [Matsui et al., 2006; Sakamoto et al., 2007] and is therefore likely to play an important role in the development of tissues other than the endoderm. On the other hand, sox 17 knockout mutant data suggest that the morphogenetic defects outside the en- doderm are secondary to defects in the endoderm [Kanai-Azuma et al., 2002].

At pre-streak stages (stage 1 and 2), sox17 expression is present either in embryonic and extraembryonic tissues of the rabbit, whereas in the mouse egg cylinder, the expression of sox 17 was first found at pre-streak stages in the extraembryonic visceral endoderm nearest to the ectoplacental cone and then to expand progressively to the whole extraembryonic visceral endoderm [Kanai-Azuma et al., 2002; Pfister et al., 2007]. Interestingly, in the chick embryo, sox17 is expressed before streak formation in early populations of posterior cells including the lower layer of Koller's sickle (KS) and in the middle layer of the posterior marginal zone (PMZ), whereas no expression was found in the epiblast layer of KS and of the PMZ and in the caudal germ wall (CGW) [Chapman et al., 2007]. In the chick, endoderm precursor cells may, thus, have descended from epiblast at a much earlier stage than in the mammalian embryo. In any case, with its early expression in the hypoblast of the AMC and the colocalization with several other anteriorizing genes such as DKK1 and Cerl [Idkowiak et al., 2004], sox17 serves to identify anterior-posterior polarity early in the pre-gastrulating rabbit embryos. Moreover, the hypoblast in the AMC could share properties with the extraembryonic endoderm of the mouse which is essential in the establishment of the body plan [Beddington and Robertson, 1999; Brennan et al., 2001; Hoodless et al., 2001; Yamamoto et al., 2001]. The expression of sox 17 in the anterior hypoblast of the AMC at pre-primitive streak formation, if compared with sox17 [Kanai-Azuma et al., 2002; Pfister et al., 2007] and hex expression in the AVE in the mouse organism [Keng et al., 1998], supports a potential role of sox17 in development of anterior structures.

In summary, the rabbit provides the tissue resolution of gene expression necessary for the analysis of the complex topography of initial endoderm formation. The current study thus extends our knowledge of mammalian germ layer generation; moreover, the rabbit's morphological similarity with most other mammalian groups during this phase of development makes the integration of the results into the concepts of early human development an intriguing proposition. Whether the identity of different parts of gut endoderm is defined by the two expression domains in the pregastrulation epiblast is now open for discussion. As a first step towards revealing the answer to this question, fate map studies should now be designed to compare directly the tissue potency in the anterior and posterior extremities of the primitive streak. 


\section{Acknowledgements}

We express our grateful acknowledgement to Kirsten FalkStietenroth, Heike Faust and Irmgard Weiss for their technical assistance and to Dr. Heiko Lickert of the Institute of Stem Cell
Research, München, Germany, for supplying the sox17 cDNA probe. This work was supported by a scholarship grant of the Syrian Government (to R.H.) and by the Deutsche Forschungsgemeinschaft (Vi 151/8-1).

\section{References}

-Alexander, J., D.Y. Stainier (1999) A molecular pathway leading to endoderm formation in zebrafish. Curr Biol 9: 1147-1157.

Beddington, R.S.P. (1981) An autoradiographic analysis of the potency of embryonic ectoderm in the 8th day postimplantation mouse embryo. J Embryol Exp Morphol 64: 87104.

Beddington, R.S.P. (1983) The origin of the foetal tissues during gastrulation in the rodent; in Johnson, M.H. (ed): Development in Mammals, vol. 5. Amsterdam, Elsevier, pp $1-32$.

Beddington, R.S.P., E.J. Robertson (1999) Axis development and early asymmetry in mammals. Cell 96: 195-209.

-Belo, J.A., T. Bouwmeester, L. Leyns, N. Kertesz, M. Gallo, M. Follettie, E.M. De Robertis (1997) Cerberus-like is a secreted factor with neutralizing activity expressed in the anterior primitive endoderm of the mouse gastrula. Mech Dev 68: 45-57.

Blum, M., P. Andre, K. Muders, A.Schweickert, A. Fischer, E. Bitzer, S. Bogusch, T. Beyer, H.W.M. van Straaten, C. Viebahn (2007) Ciliation and gene expression distinguish between node and posterior notochord in the mammalian embryo. Differentiation 75 : 133-146.

Bogue, C.W., G.R. Ganea, E. Sturm, R. Ianucci, H.C. Jacobs (2000) Hex expression suggests a role in the development and function of organs derived from foregut endoderm. Dev Dyn 219: 84-89.

Brennan, J., C.C.Lu, D.P. Norris, T.A. Rodriguez, R.S.Beddington, E.J. Robertson (2001) Nodal signalling in the epiblast patterns the early mouse embryo. Nature 411: 965-969.

-Chalmers, A.D., J.M. Slack (2000) The Xenopus tadpole gut: fate maps and morphogenetic movements. Development 127: 381-392.

-Chapman, S.C., F.R. Schubert, G.C. Schoenwolf, A. Lumsden (2003) Anterior identity is established in chick epiblast by hypoblast and anterior definitive endoderm. Development 130: 5091-5101.

-Chapman, S.C., K. Matsumoto, Q. Cai, G.C. Schoenwolf (2007) Specification of germ layer identity in the chick gastrula. BMC Dev Biol 30: 91.

Denker, H.-W. (2000) Structural dynamics and function of early embryonic coats. Cells Tissues Organs 166: 180-207.
Gardner, R.L., J. Rossant (1979) Investigation of the fate of 4.5 day post-coitum mouse inner cell mass cells by blastocyst injection. J Embryol Exp Morphol 52: 141-152.

Hoodless, P.A., M. Pye, C. Chazaud, E. Labbe, L. Attisano, J. Rossant, J.L. Wrana (2001) FoxH1 (Fast) functions to specify the anterior primitive streak in the mouse. Genes Dev 15: 1257-1271.

Hudson, C., D. Clements, R.V. Friday, D. Stott, H.R. Woodland (1997) Xsox17alpha and -beta mediate endoderm formation in Xenopus. Cell 91: 397-405.

Idkowiak, J., G. Weisheit, J. Plitzner, C. Viebahn (2004) Hypoblast controls mesoderm generation and axial patterning in the gastrulating rabbit embryo. Dev Genes Evol 214: 591605.

Kadokawa, Y., Y. Kato, G. Eguchi (1987) Cell lineage analysis of the primitive and visceral endoderm of mouse embryos cultured in vitro. Cell Diff 21: 69-76.

Kanai, Y., M. Kanai-Azuma, T. Noce, T.C. Saido, T. Shiroishi, Y. Hayashi, K. Yazaki (1996) Identification of two Sox17 messenger RNA isoforms, with and without the high mobility group box region, and their differential expression in mouse spermatogenesis. J Cell Biol 133: 667-681.

Kanai-Azuma, M., Y. Kanai, J.M. Gad, Y. Tajima, C. Taya, M. Kurohmaru, Y. Sanai, H. Yonekawa, K. Yazaki, P.P. Tam, Y. Hayashi (2002) Depletion of definitive gut endoderm in Sox17-null mutant mice. Development 129: 2367-2379.

Keller, R.E. (1975) Vital dye mapping of the gastrula and neurula of Xenopus laevis. I. Prospective areas and morphogenetic movements of the superficial layer. Dev Biol 42: 222-241.

Keller, R.E. (1976) Vital dye mapping of the gastrula and neurula of Xenopus laevis. II. Prospective areas and morphogenetic movements of the deep layer. Dev Biol 51: 118-137.

Keng, V.W., K.E. Fujimori, Z. Myint, N. Tamamaki, Y. Nojyo, T. Noguchi (1998) Expression of Hex mRNA in early murine postimplantation embryo development. FEBS Lett 426: 183-186.

Kimura, W., S. Yasugi, C.D. Stern, K. Fukuda (2006) Fate and plasticity of the endoderm in the early chick embryo. Dev Biol 289: $283-$ 295.
Kimura, W., S. Yasugi, K. Fukuda (2007) Regional specification of the endoderm in the early chick embryo. Dev Growth Differ 49: 365372.

Kinder, S.J., T.E. Tsang, S.L.Ang, R.R. Behringer, P.P. Tam (2001) Defects of the body plan of mutant embryos lacking Lim1, Otx2 or Hnf3beta activity. Int J Dev Biol 45: 347355.

Kirby, M.L., A. Lawson, H.A. Stadt, D.H. Kumiski, K.T. Wallis, E. McCraney, K.L. Waldo, Y.X. Li, G.C. Schoenwolf (2003) Hensen's node gives rise to the ventral midline of the foregut: implications for organizing head and heart development. Dev Biol 253: 175188.

Kubo, A., K. Shinozaki, J.M. Shannon, V. Kouskoff, M. Kennedy, S. Woo, H.J. Fehling, G. Keller (2004) Development of definitive endoderm from embryonic stem cells in culture. Development 131: 1651-1662.

Kwon, G.S., M. Viotti, A.K. Hadjantonakis (2008) The endoderm of the mouse embryo arises by dynamic widespread intercalation of embryonic and extraembryonic lineages. Dev Cell 15: 509-520.

Lawson, K.A. (1999) Fate mapping the mouse embryo. Int J Dev Biol 43: 773-775.

Lawson, K.A., J.J. Meneses, R.A. Pedersen (1986) Cell fate and cell lineage in the endoderm of the presomite mouse embryo, studied with an intracellular tracer. Dev Biol 115: $325-$ 339.

Lawson, K.A., J.J. Meneses, R.A. Pedersen (1991) Clonal analysis of epiblast fate during germ layer formation in the mouse embryo. Development 113: 891-911.

Lawson, K.A., R.A. Pedersen, S. Van de Geer (1987) Cell fate, morphogenetic movement and population kinetics of embryonic endoderm at the time of germ layer formation in the mouse. Development 101: 627-652.

Lawson, K.A., R.A. Pedersen (1992) Clonal analysis of cell fate during gastrulation and early neurulation in the mouse; in Chadwick, D.J., J. Marsh (eds) Postimplantation Development in the Mouse. Wiley, Chichester (CIBA Foundation Symp 165), pp 3-26.

Lawson, KA., G.C. Schoenwolf (2003) Epiblast and primitive-streak origins of the endoderm in the gastrulating chick embryo. Development 130: 3491-3501. 
Lefebvre, V., B. Dumitriu, A. Penzo-Mendez, Y. Han, B. Pallavi (2007) Control of cell fate and differentiation by Sry-related high-mobilitygroup box (Sox) transcription factors. Int J Biochem Cell Biol 39: 2195-2214.

-Levak-Svajger, B., A. Svajger (1974) Investigation on the origin of the definitive endoderm in the rat embryo. J Embryol Exp Morphol 32: 445-459.

Lin, T.P., P.A. Labosky, L.B. Grabel, C.A. Kozak, J.L. Pitman, J. Kleeman, C.L. MacLeod (1994). The Pem homeobox gene is X-linked and exclusively expressed in extraembryonic tissues during early murine development. Dev Biol 166: 170-179.

Lowe, L.A, D.M. Supp, K. Sampath, T. Yokoyama, C.V.E. Wright, S.S. Potter, P. Overbeek, M.R. Kuehn (1996) Conserved left-right asymmetry of nodal expression and alterations in murine situs inversus. Nature 381: 158-161.

Matsui, T., M. Kanai-Azuma, K. Hara, S. Matoba, R. Hiramatsu, H. Kawakami, M. Kurohmaru, P. Koopman, Y. Kanai (2006) Redundant roles of Sox17 and Sox18 in postnatal angiogenesis in mice. J Cell Sci 119: 3513-3526.

Mossman, H.W. (1937) Comparative morphogenesis of the fetal membranes and accessory uterine structures. Contrib Embryol Carnegie Inst 26: 129-246.

Pander, C.H. (1817) Beiträge zur Entwicklungsgeschichte des Hühnchens im Eye. Brönner, Würzburg.

- Parameswaran, M., P.P.L. Tam (1995) Regionalisation of cell fate and morphogenetic movement of the mesoderm during mouse gastrulation. Dev Genet 17: 16-28.

- Pennisi, D., J. Gardner, D. Chambers, B. Hosking, J. Peters, G. Muscat, C. Abbott, P. Koopman (2000) Mutations in Sox18 underlie cardiovascular and hair follicle defects in ragged mice. Nat Genet 24: 434-437.
Pfister, S., K.A. Steiner, P.P.L. Tam (2007) Gene expression pattern and progression of embryogenesis in the immediate post-implantation period of mouse development. Gene Expr Patterns 7: 558-573.

Quinlan, G.A., E.A. Williams, S.S. Tan, P.P.L. Tam (1995) Neuroectodermal fate of epiblast cells in the distal region of the mouse egg cylinder: implication for body plan organization during early embryogenesis. Development 121: 87-98.

Sakamoto, Y., K. Hara, M. Kanai-Azuma, T. Matsui, Y. Miura, N. Tsunekawa, M. Kurohmaru, Y. Saijoh, P. Koopman, Y. Kanai (2007) Redundant roles of Sox17 and Sox18 in early cardiovascular development of mouse embryos. Biochem Biophys Res Commun 360: 539-544.

Skreb, N., A. Svajger, B. Svajger (1976) Developmental potentialities of the germ layers in the mammals. CIBA Found Symp 40. Chichester, Wiley, pp 27-39.

Stainier, D.Y. (2002) A glimpse into the molecular entrails of endoderm formation. Genes Dev 16: 893-907.

Tam, P.P.L. (1989) Regionalisation of the mouse embryonic ectoderm: allocation of prospective ectodermal tissues during gastrulation. Development 107: 55-67.

Tam, P.P.L., R.S.P. Beddington (1987) The formation of mesodermal tissues in the mouse embryo during gastrulation and early organogenesis. Development 99: 109-126.

Tam, P.P.L, R.S.P. Beddington (1992) Establishment and organization of germ layers in the gastrulating mouse embryo; in Chadwick, D.J., J. Marsh (eds): Postimplantation Development in the Mouse. CIBA Foundation Symp 165. Chichester, Wiley, pp 27-49.
Tam, P.P., P.L. Khoo, S.L. Lewis, H. Bildsoe, N. Wong, T.E. Tsang, J.M. Gad, L. Robb (2007) Sequential allocation and global pattern of movement of the definitive endoderm in the mouse embryo during gastrulation. Development 134: 251-260.

Tam, P.P., E.A. Williams, W.Y. Chan (1993) Gastrulation in the mouse embryo: ultrastructural and molecular aspects of germ layer morphogenesis. Microsc Res Tech 26: 301328.

Viebahn, C. (2004) Gastrulation in the rabbit; in Stern, C.D.S. (ed): Gastrulation. Cold Spring Harbor, Cold Spring Habor Laboratory Press, pp 263-274.

Viebahn, C., C. Stortz, S.M. Mitchell, M. Blum (2002) Low proliferative and high migratory activity in the area of Brachyury expressing mesoderm progenitor cells in the gastrulating rabbit embryo. Development 129: 23552365.

-Warga, R.M., C. Nüsslein-Volhard (1999) Origin and development of the zebrafish endoderm. Development 126: 827-838.

Weisheit, G., K.D. Mertz, K. Schilling, C. Viebahn (2002) An efficient in situ hybridisation protocol for multiple tissues sections and probes on miniaturized slides. Dev Genes Evol 212: 403-406.

Wood, H.B., V. Episkopou (1999) Comparative expression of the mouse Sox 1 , Sox 2 and Sox 3 genes from pre-gastrulation to early somite stages. Mech Dev 86: 197-201.

Woodland, H.R., A.M. Zorn (2008) The core endodermal gene network of vertebrates: combining developmental precision with evolutionary flexibility. Bioessays 30: 757-765

Yamamoto, M., C. Meno, Y. Sakai, H. Shiratori, K. Mochida, Y. Ikawa, Y. Saijoh, H. Hamada (2001) The transcription factor FoxH1 (FAST) mediates Nodal signaling during anterior-posterior patterning and node formation in the mouse. Genes Dev 15: 1242 1256. 